\title{
The most important factors of causing hearing loss following central auditory system disorder and central nervous system (CNS) disorder
}

\author{
Abstract \\ Hearing system is a bottom up and top - down processing system. Dysfunction in peripheral \\ hearing system may cause dysfunction in central hearing system but the question is whether the \\ central auditory system disorder or central nervous system disorder induce the hearing loss? \\ We deal with this subject in this paper by: \\ a) Contralateral Sensory Neural Hearing Loss (S.N.H.L) following Ipsilateral Central \\ Auditory Processing Disorder (CAPD) \\ b) Mentioning studies on animals \\ c) The correlation between Limbic system and Hearing system \\ d) Neurotransmitters role in Hearing system \\ e) Sudden Deafness following infarctions, anti depression and addictive drugs, \\ contraceptive pill, diabetes, stress, multiple sclerosis. \\ f) Some of the CNS disorders which cause hearing loss presumably due to dysfunction \\ in Neurotransmitters. \\ g) The correlation between Meniere, Otosclerosis, Auditory Neuropathy, \\ Temporomandibular Joint Disorders with CNS.
}

Volume 2 Issue 6 - 2015

\author{
Alireza Bina \\ Atieh Hospital,Audiology Clinic, Iran
}

Correspondence: Alireza Bina,Atieh Hospital,Audiology Clinic, Iran, Tel 817-666-2926,Email bina_alireza@yahoo.com

Received: January 27, 20I5 | Published: July 18, 2015
Abbreviations: CAPD, central auditory processing disorder; SNHL, sensory neural hearing loss; SSP, sub acute sclerosing panencephalitis; OME, otitis media with effusion; MS, multiple sclerosis; TMJD, temporomandibular joint disorders

\section{Discussion}

One of the etiology of Central Auditory Processing Disorder (CAPD) is Peripheral Hearing Loss, but does Central auditory system disorder cause hearing loss? Mild lesions in Central Auditory Pathways may not cause hearing loss (depends on the site and extent of lesion) or cause slight hearing loss.

\section{Case report}

A seven years old child whose mother complained of her child speech recognition in noisy environment referred to our clinic. The child was Autistic and the Audiometry and Tympanometry revealed normal peripheral hearing in both ears but probably child suffered from CAPD. Experience has shown that in those patients who have Sensory Neural Hearing Loss (S.N.H.L), probably due to CNS disorder or central auditory system disorder, high frequency hearing loss is less common but flat sensorineural hearing loss, Low tone loss, slight notch in $1 \mathrm{KHZ}$ or $2 \mathrm{KHZ}$ (slight hearing loss 20-30 dB HL only in 1 or $2 \mathrm{KHZ}$ and normal hearing in other frequencies) which have been seen in patients using anti depression drugs or having psychiatry disorders, is much more common. Normal Audiogram (normal peripheral hearing) with better hearing at $2 \mathrm{KHZ}$ compare to the other frequencies has been seen in Autistic patients. But what about large lesions in central auditory system? Large lesions in central auditory system may cause remarkable hearing loss. In a study over Dogs, after lesions of the brachium of the inferior colliculus and medial geniculate, hearing loss was seen. ${ }^{1}$ Damage to the primary auditory cortex in human leads to a loss of any awareness of sound (depends on the extent of lesion), but an ability to react reflexively to sounds remains as there is a great deal of subcortical processing in the auditory brain stem and mid brain..$^{2,3}$

\section{Contra lateral S.N.H.L following ipsilateral CAPD}

Unilateral damage in the primary Auditory Cortex likely cause contra lateral S.N.H.L. ${ }^{4}$ It seems that ipsilateral dysfunction in other Central Auditory sections such as Inferior Colliculus may cause contra lateral S.N.H.L.. ${ }^{5}$ Contralateral S.N.H.L is common in patients who is affected unilaterally S.N.H.L. ${ }^{6,7}$ Suddenly contra lateral S.N.H.L is occurred in patients who have unilaterally hearing loss. ${ }^{8}$ Is sensory deprivation in ipsilateral Central Auditory system by unilateral hearing loss involved in causing contra lateral hearing loss?., ${ }^{910}$ However, contra lateral hearing loss could be occurred because of other reasons .So evaluation and if it would be necessary therapy of Central Auditory system in non affected ear in patients who have idiopathic unilaterally suddenly S.N.H.L or unilaterally hearing loss is essential Improvement of Tinnitus in contra lateral (non implanted ear) has been seen in patients who have bilaterally profound S.N.H.L and undergone of unilaterally Cochlear Implant. ${ }^{11}$ The reason of this feature is unknown, but could stimulation of ipsilateral Central Auditory system by unilateral Cochlear Implant cause improvement of Tinnitus in the non implanted ear? Whereas there may be another reason for this feature.

\section{The correlation between limbic system and hearing system}

There is a significant relationship between limbic disorders and hearing impairment. In a study an acute limbic - cerebellar syndrome 
was seen in six industrial workers who inhaled Trimethyltin (white solid which is highly toxic). Clinical features included hearing loss, amnesia, disorientation, confabulation and so on. ${ }^{12}$

Limbic-thalamus: Thalamus disorder could cause hearing loss, specifically mass lesion relative to Gray matter in the thalamus. ${ }^{13}$

Limbic - hypothalamus: Hypothalamus may be involved in Meniere's disease which will be described later.

Limbic - hippocampus: Age -related hearing loss (Presbycusis) is accompanied by the degeneration of synapses in the hippocampus ${ }^{14}$. High intensity noise exposure not only damages the Cochlea but also causes a significant and persistent decrease in the hippo campal neuro genesis that may contribute to functional deficits in memory ${ }^{15}$. Other disabilities such as ADHD, AUTISM, DYSLEXIA and Learning Disability are common in children who suffer from sensorineural hearing loss. Hippocampus is involved in some of these pathologies. ${ }^{16,17}$ Bilateral loss of Vestibular function is associated with a significant bilateral atrophy of the Hippocampus, which correlated with the patients spatial memory deficits. Patients who were recovered from unilateral Vestibular Neuritis have been reported to exhibit a significant atrophy of the left posterior Hippocampus. ${ }^{18}$ So supposedly there is a correlation between Hippocampus and Vestibular, Auditory system.

Limbic- Amygdala: Bilateral amygdale lesions may cause auditory recognition of fear and anger. ${ }^{19}$

Limbic - Cingulategyrus: FMRI study shows that left posterior cingulategyrusis responsible for distinguish of threat-related words. ${ }^{20}$

Limbic - Basal ganglia: In a study (case report) hearing loss occurred following infraction of basal ganglia. ${ }^{21}$ Basal ganglia has well connected with the cerebral cortex, thalamus and other brain areas.

\section{Neuro transmitters role in hearing system}

Brain neurotransmitters are involved in hearing system (GlutamateGABA-Serotonin-Acetylcholine- Dopamine-Nor epinephrine and etc). It seems that any dysfunction in these Neurotransmitters (increasing or decreasing from normal range) may cause hearing loss and also hearing loss could cause dysfunction in neurotransmitters in the brain. Neurotransmitters levels change in stress, diabetes, Presbycusis, depression, some CNS disorders ,taking anti depression, addictive drugs and contraceptive pill, which these changes could impact peripheral and central hearing systems.

\section{Some of the CNS disorders which cause hearing loss}

Essential tremor: Essential tremor is a degenerative disorder of the CNS which most recognizable feature is a tremor of the arms or hands during voluntary movements such as eating and writing. S.N.H.L. is more common in this pathology than Parkinson disease probably due to dysfunction in neurotransmitters particularly GABA. ${ }^{22}$

Case report: A 45years old male patient who suffered from essential tremor referred to our clinic by ENT physician. He had no other diseases such as diabetes, hypertension, anxiety, depression or any experience of working in noisy environment also he did not take any medicines for Essential Tremor. The hearing result was bilaterally S.N.H.L. with SRT $=45 \mathrm{dBHL}$ and good SDS.

Wernicke encephalopathy: Wernicke Encephalopathy mentions the presence of neurological symptoms caused by biochemical lesions of the CNS after depletion of B vitamin reserves, in particular Thiamine, or by its difficulties in accessing the mitochondria. One of the causes of S.N.H.L. in Wernicke Encephalopathy is probably due to alter the glutamate uptake of astrocytes glial cells of the brain leading to Excitotoxicity.

Myasthenia gravis: Myasthenia gravis is an autoimmune neuromuscular disease that causes fluctuating muscle weakness and fatigability. It is an autoimmune disorder in which weakness is caused by circulating anti bodies that hinder acetylcholine receptors at the post synaptic neuromuscular junction, inhibiting the excitatory effects of the neurotransmitters acetylcholine on nicotinic receptors throughout neuromuscular junctions. Acetylcholine is the chief neurotransmitter of the efferent auditory pathways. Dysfunction of this neurotransmitter may cause cochlear damage in Myasthenia gravis disease. ${ }^{23}$

\section{The correlation between meniere,otosclerosis,} auditory neuropathy, temporomandibular joint disorders with CNS

Otosclerosis disease and CNS: One of the etiology of Otosclerosis disease is measles virus .Otosclerosis is association with primary Sub acute Sclerosing Panencephalitis (SSP). SSP is rare chronic, progressive encephalitis caused by Measles Virus, a defective measles virus infection of the central nervous system and leads to positive immuno reaction in areas of active Otosclerosis. Medications which are used for the treatment of SSP such as antiviral Medications may be useful for the treatment of Otosclerosis and evaluation of Measles Virus in the serum of patients which therapists suspicious to Otosclerosis can be used as one of the tests beside other tests for differential diagnosis between Otosclerosis and similar pathologies such as Superior Semicircular Canal Dehiscence(SSCD), Tympano sclerosis, Ossicular Chain and so on ,because Measles Virus is involved in Otosclerosis and may not involve in these pathologies. Otosclerosis has been reported in CNS pathologies such as Fabry's disease. One of the features of this disease is reddish purple blemishes as the same as Measles. However, Measles is not probably implicated in Fabre disease. ${ }^{24-26}$

Meniere's disease and CNS: In Meniere's patients neuro endocrine logical pathways (Hypothalamic-pituitary -adreno cortical axis) controlling stress can be disrupted probably due to decline of hypothalamic dopaminergic inhibition of prolactin secretion. Increasing cortisol levels impact the endo lymphatic homeostasis leads to a deterioration of hearing at high frequencies (particularly $2 \mathrm{KHZ}$ ) in the early stage of Meniere's disease. However, many Meniere's patients have low tone loss in the beginning the disease. ${ }^{27,28}$ According to these finding Dopamine Agonist Medications may be useful for the treatment of Meniere's disease.

Case report: A female patient who had suffered from Meniere's disease referred to our office ten years ago. She had slight hearing loss at high frequency ( 2 and $4 \mathrm{KHZ}=20 \mathrm{~dB} \mathrm{HL}$ ) in her left ear with severe vertigo but now she has flat S.N.H.L. with $S R T=40 \mathrm{~dB} H L$ in left ear and vertigo attacks has been reduced (patient said) comparing to ten years ago that she had slight high frequency hearing loss.

Auditory neuropathy and CNS: CNS pathologies could cause Auditory Neuropathy such as Brain stem de myelination, multiple sclerosis and crypto coccal CNS infection. Auditory Neuropathy could also cause CAPD. ${ }^{29-31}$

Temporomandibular joint disorders (TMJD) and CNS: It sounds that there is a relationship between TMJD and CNS. There is some evidence which indicated that hyperactivity of jaw closing muscles 
may originate in the CNS. TMJD also could cause sensorineural hearing loss and conductive hearing loss (otitis media), so it should be considered in diagnosis of idiopathic suddenly sensory neural hearing loss, sensory neural hearing loss and Otitis Media with Effusion (OME). Tinnitus, vertigo and otalgia are the other otologic symptoms of TMJD. ${ }^{32-37}$

Suddenly hearing loss following infarctions: One of the major labyrinthine artery is anterior inferior cerebellar artery. Infarction of this artery could cause suddenly S.N.H.L. ${ }^{38}$ Whereas infarction of other parts of brain may cause hearing loss such as bilateral temporal embolism and Basal ganglia infarction which has been mentioned before.

Suddenly S.N.H.L. following anti depression and addictive drugs, contraceptive pill: There is a significant relationship between depression and hearing impairment. Anti depression and addictive drugs also could cause suddenly S.N.H.L. For example suddenly hearing loss has been reported pursuant to Fluvoxamine and Mirtazapine therapy ${ }^{39,40}$ Fluvoxamine is a serotonin reuptake inhibitor. (It increases serotonin) Mirtazapine is a Norepinephrine Serotonin reuptake inhibitor. (It increases Nor epinephrine-Serotonin) Addictive drugs also cause suddenly hearing loss.

Case report: A 25years old male patient who suffered from bilaterally sudden S.N.H.L. following using Crack (Drug abuse).The hearing result was bilateral S.N.H.L. with SRT $=80 \mathrm{HL}$ and poor SDS. The patient did not have any experience of working in noisy environment, hypertension, diabetes, or any other diseases. Crack initial effect is to release a large amount of Dopamine. Although anti depression and addictive drugs have different side effects such as hypertension which may cause lesion in peripheral hearing system, but changing Neurotransmitters levels in the brain may be one of the reasons of hearing loss in these patients. Contraceptive pill cause suddenly S.N.H.L probably due to changes in neurotransmitter receptor concentrations. ${ }^{41}$

\section{Suddenly hearing loss following multiple sclerosis (MS)}

MS (Multiple Sclerosis) disease could be causing suddenly S.N.H.L. In a study unilaterally sudden deafness occurred in two patients who had MS with lesions in the pantone and medulla oblongata both are situated in the brain stem. ${ }^{42}$

The correlation between diabetes and stress with S.N.H.L: S.N.H.L. and suddenly S.N.H.L. are common in diabetes and patients who suffer from stress. Diabetes causes damage in the CNS and Peripheral system. One of the reasons of S.N.H.L.in diabetes patients is Micro angiopathic disease in the inner ear which causes S.N.H.L. particularly high tone loss. High tone loss is a typical shape of hearing loss in diabetes patients, but diabetes may cause S.N.H.L. because of damage in the CNS(typically in the Hippocampus and cortex)specifically in those patients who have low tone loss or flat S.N.H.L. ${ }^{43,44}$ Stress changes catecholamine's 11 (Dopamine ,Nor epinephrine, Epinephrine) at blood and resulted to the exaggeration of cochlear sympathetic innervation and S.N.H.L. ${ }^{45,46}$

Case report: A 45years old male diabetic patient who had experience of sudden deafness in his left ear two years ago, referred to our clinic immediately after sensation of Tinnitus and suddenly hearing loss in his right ear without complaining of dizziness or vertigo. The patient had no experience of working in noisy environment or hypertension, stress, anxiety, depression and other diseases except diabetes. Tympanometry and Otoscopy revealed normal middle ear function in both ears. Acoustic Reflex was absent in both ears (IPSI and CONTRA). Audiometry indicated severe to profound flat S.N.H.L. in both ears with poor SDS. Imaging results was normal. The patient underwent of intra tympanic steroid therapy in his right ear. Unfortunately after 3 sessions of injection no improvement of hearing was seen.

Suddenly hearing loss following stress (case report): A 55years old male patient who was cardio surgeon referred to our clinic immediately after filling Tinnitus and suddenly hearing loss in his right ear following stress. He had experience of sudden deafness in his left ear some years ago .He had no complaints of dizziness or vertigo and did not have hypertension, diabetes or any other diseases. The patient suffered from sleep disorder following suddenly hearing loss too, which probably indicated dysfunction in Neurotransmitters. Otoscopy and Tympanometry revealed normal middle ear function in both ears. Acoustic reflex was absent in both ears. (IPSI and CONTRA) Audiometry suggested bilateral severe to profound S.N.H.L with poor SDS. MRI result was normal. The patient underwent of intra tympanic steroid therapy. After two sessions of injection, hearing loss in right ear returned to near normal hearing but hearing improvement was not stable and hearing returns to severe to profound hearing loss with poor SDS in right ear. If suddenly hearing loss in this patient was occurred because of dysfunction in Neurotransmitters, could medications which regulate Neurotransmitters level be helpful to improve hearing loss? Further investigations need to determine this subject.

\section{Conclusion}

We hear with our brain the same as our ears. There is a significant relationship between brain disorders and hearing impairment. Not only dysfunction in central auditory system may cause hearing impairment, but also dysfunction in non auditory system in the brain may impact on hearing system. There are many theories for idiopathic suddenly S.N.H.L, viral, ischemia, Autoimmune diseases and so on, but we suggested that dysfunction in Neurotransmitters as another hypothesis in suddenly S.N.H.L. Neurotransmitters have important role in hearing system, so dysfunction in Neurotransmitters should be considered in diagnosis and treatment of hearing loss. Evaluation of Neurotransmitters in the blood, cochlea and brain is possible with technologies included of high - performance liquid chromatography, electro chemical detection, MR-Spectroscopy, in vivo micro dialysis. ${ }^{47}$ There are several Neurotransmitters which are involved in hearing system, but we deal with some of them in this study. Dysfunction in Peripheral hearing system may cause dysfunction in Central Auditory system and dysfunction in Central Auditory system may impact on peripheral hearing system. Most of the diseases such as Otosclerosis and Meniere's diseases which are peripheral pathologies originate from CNS. There is always a reason for hearing loss. Even Presbycusis has different types depends on site and type of lesion. Finding out the reason of hearing loss helps therapist for treatment or at least prevent of hearing impairment.

\section{Acknowledgments}

None.

\section{Conflicts of interest}

Author declares there are no conflicts of interest.

\section{Funding}

None. 


\section{References}

1. Heffner RS, Heffner HE. Hearing Loss in dogs after lesions of the brachium of the inferior colliculus and medial geniculate. J Comp Neurol. 1984;230(2):207-217.

2. Heffner HE, Heffner RS. Hearing Loss in Japanese macaques following bilateral auditory cortex lesions. J Neurophysiol. 1986;55(2):256-271.

3. Brody RM, Nicholas BD, Wolf MJ, et al. Cortical deafness: A case report and review of the literature. Otol Neurotol. 2013;48(1):35-49.

4. Heffner HE, Heffner RS. Unilateral auditory cortex ablation in macaques results in a contralateral hearing loss. $J$ Neurophysiol. 1989;62(3):789-801.

5. Strauss C, Naraghi R, Bischoff BJ, et al. Contralateral hearing loss as an effect of venous congestion at the ipsilateral inferior colliculus after micro vascular decompression: report of a case. J Neurol Neurosurg Psychiatry. 2000;69(5):679-682.

6. Uwiera TC, DeAlarcon A, Meinzen-Derr J, et al. Hearing loss progression and contralateral involvement in children with unilateral sensorineural hearing loss. Ann Otol Rhinol Laryngol. 2009;118(11):781-785.

7. Akinobu K, Hiroaki N, Taizo T . Hearing changes in the contralateral ear with juvenile unilateral profound hearing loss of unknown origin International Journal of Pediatric Otorhinolaryngology Extra. 2006;1(3):192-195.

8. Walsh RM, MuPunt JA, O Donoghue GMrty GE. Sudden contralateral deafness following cerebellopontine angle tumor surgery. Am J Otol. 1994;15(2):244-246.

9. Syka J. Plastic changes in the Central Auditory System After Hearing Loss, Restoration of Function, and During Learning . Physiol Rev. 2002;82(3):601-636.

10. Kotak VC, Fujisawa S, Lee FA, et al. Hearing Loss Raises Excitability in the Auditory Cortex. J Neurosci. 2005;25(13):3908-3918.

11. Quaranta N, Fernandez-Vega S, D'elia C, et al.The effect of unilateral multichannel cochlear implant on bilaterally perceived tinnitus. Acta Otolaryngol. 2008;128(2):159-163.

12. Besser R, Krämer G, Thümler R, et al. Acute trimethyltinlimbliccerebellar syndrome. Neurology. 1987;37(6):945-50.

13. Kaneko T, Kawakami R, Fujinaga Y, et al. Degenerative Thalamic Hamartoma CT and MR Imaging Features. AJNR Am J Neuroradiol. 2004;25(5):766-768

14. Yu YF, Zhai F, Dai CF, et al.The relationship between age-related hearing loss and synaptic changes in the hippocampus of C57 BL /6. J Exp Gerontol. 2011;46(9):716-722.

15. Kraus KS, Mitra S, Jimenez Z, et al. Noise trauma impairs neurogenesis in the rat hippocampus. Neuroscience. 2010;167(4):1216-1226.

16. Plessen KJ, Bansal $\mathrm{R}, \mathrm{Zhu} \mathrm{H}$, et al. Hippocampus and amygdala morphology in attention-deficit/hyperactivity disorder. Arch Gen Psychiatry. 2006;63(7):795-807.

17. Groen W, Teluij M, Buitelaar J, et al.Amygdala and hippocampus enlargement during adolescence in autism. $J$ Am Acad Child Adolesc Psychiatry. 2010;49(6):552-560.

18. Phoebe Chung, Catherine Gliddon, Ming Zhang, et al. The effects of bilateral vestibular loss on hippo campal volume, neuronal number, and cell proliferation in rats. Front Neurol. 2012;3:20.

19. Scott SK, Young AW, Calder AJ, et al. Impaired Auditory recognition of fear and anger following bilateral amygdale lesions. Nature. 1997;385(6613):254-257.

20. Maddock RJ, Buonocore MH. Activation of left posterior cingulate gyrus by the auditory presentation of threat-related words an FMRI study. Psychiatry Res. 1997;75(1):1-14.
21. Russo LS Jr. Focal Dystonia and lacunal Infarction of the Basal Ganglia. Arch Neurol. 1983;40(1):61-62.

22. Ondo WG, Sutton L, Dat Vuong K, et al.Hearing impairment in essential tremor. Neurology. 2003;61(8):1093-1097.

23. Hamed SA, Elattar AM, Hamed EA. Irreversible cochlear damage in myasthenia gravis_otoacoustic emission analysis. Acta Neurol Scand. 2006;113(1):46-54.

24. McKenna MJ, Mills BG. Ultra structural and immunohistochemical evidence of measles virus in active Otosclerosis. Acta Otolaryngol Suppl. 1990;470:130-139.

25. Fellgiebel A, Müller MJ, Ginsberg L. CNS manifestations of Fabry's disease. Lancet Neurol. 2006;5(9):791-795.

26. Felisati G, Salvatici E, Pipolo C, et al. Fabry disease presenting with sudden hearing loss and otosclerosis: a case report. J Med Case Rep. 2012;6:112.

27. Aoki M, Wakaoka Y, Hayashi H, et al. The relevance of hypothalamuspituitary-adernocortical axis-related hormones to the cochlear symptoms in meniere's disease. Int J Audiol. 2011;50(12):897-904.

28. Horner KC, Cazals Y. Stress in hearing and balance in meniere's disease. Noise Health. 2003;5(20):29-34.

29. Mallur PS, Lalwani AK. Fluctuating corticosteroid responsive auditory neuropathy/dys synchrony is suggestive of central nervous system pathology. Otol Neurotol. 2007;28(8):1002-1004.

30. Celis-Aguilar E, Macias-Valle L, Coutinho-De Toledo H. Auditory neuropathy secondary to cryptococcal central nervous system infection in 2 immuno compromised patients. Otolaryngol Head Neck Surg. 2012;147(3):597-598.

31. Joseph P Pillion. Speech Processing Disorder in Neural Hearing Loss. Case Reports in Medicine. 2012;7.

32. Yemm R. Neurophysiologic studies of temporomandibular joint dysfunction. Oral Sci Rev. 1976;7:31-53.

33. Axelsson R, Tullberg M, Ernberg M, et al. Symptoms and signs of temporomandibular disorders in patients with sudden sensorineural hearing loss. Swed Dent J. 2009;33(3):115-123.

34. Baldursson G, Blackmer ER. Temporomandibular joint symptoms in patients with mid frequency sensorineural hearing loss. Ear Hear. 1987;8(2):63-67.

35. Bubon MS. Documented instance of restored conductive hearing loss. Funct Orthod. 1995;12(1):26-29.

36. Youniss S. The relationship between craniomandibular disorders and otitis media in children. Cranio. 1991;9(2):169-173.

37. Okeson, Jeffrey P. Management of temporomandibular disorders and occlusion.

38. Lee H, Whitman GT, Lim JG, Lee SD, Park YC (2001) Bilateral Sudden Deafness as a prodrome of Anterior Inferior Cerebellar Artery Infarction. Arch Neurol. 2003;58(8):1287-1289.

39. Engin Dursun, Abdullah Akpinar, Bilal Battal . Sudden hearing loss Associated with mirtazapine therapy: a case report. Bulletin of clinical psychopharmacology. 2009;19(4):417-419.

40. Duru Gundogar, Fevzi sefa Derekoy, Deniz Yilmaz. Hearing loss and tinnitus during fluvoxamine treatment: a case report. $B C P$. 2009;19(1):59-63.

41. Strachan D. Sudden sensorineural deafness and hormone replacement therapy. J Laryngol Otol. 1996;110(12):1148-1150.

42. Bozidar D, Ksenija RJ, Vlademir SK, et al.Sudden hearing loss at the initial monosymptom of multiple sclerosis. Neurology. $1993 ; 43(12): 2703$ 
43. Trudeau F, Gagnon S, Massicotte G. Hippocampal synaptic plasticity and glutamate receptor regulation influences of diabetes mellitus. Eur J Pharmacol. 2004;490(1-3): 177-186.

44. Bree AJ, Puente EC, Daphna-Iken D, et al. Diabetes increases brain damage caused by severe hypoglycemia. AMJ Physiol Endocrinol metab. 2009;297(1):E194-E201.
45. Schmitt C, Patak M, Kroner-Herwig B. Stress and the onset of sudden hearing Loss and tinnitus. Int Tinnitus J. 2000;6(1):41-49.

46. Muchnik C, Hildesheimer M, Rubinstein M. Effect of Emotional stress on Hearing. Arch Otorhinolaryngol. 1980;228(4):295-298.

47. Rarey KE, Ross MD, Smith CB. Quantitative evidence for cochlear, non-neuronal norepinephrine. Hear Res. 1981;5(1):101-108. 